\title{
A Comparative Study of Resonances in the Dynamics of the Mimas-Tethys System
}

\author{
Hemant Kumar Mishra1, Govind Kumar Jha'², Sarita Jha ${ }^{3}$ \\ ${ }^{1}$ Department of Mathematics, Chatra College, Chatra, India \\ ${ }^{2}$ Department of Mathematics, Vinoba Bhave University, Hazaribag, India \\ ${ }^{3}$ Department of Mathematics, K. B. Women's College, Hazaribag, India \\ Email:hemumishra@gmail.com,jhagovi@gmail.com, saritajhakbw.vbu@gmail.com
}

How to cite this paper: Mishra, H.K., Jha, G.K. and Jha, S. (2017) A Comparative Study of Resonances in the Dynamics of the Mimas-Tethys System. International Journal of Astronomy and Astrophysics, 7, 28-37. https://doi.org/10.4236/ijaa.2017.71003

Received: December 13, 2016

Accepted: March 18, 2017

Published: March 21, 2017

Copyright $\odot 2017$ by authors and Scientific Research Publishing Inc. This work is licensed under the Creative Commons Attribution International License (CC BY 4.0).

http://creativecommons.org/licenses/by/4.0/ (c) (i) Open Access

\begin{abstract}
The probability of capture of Mimas-Tethys in 2:4 resonance was found to be 0.76 by Champenois when they considered the orbit of Tethys to be elliptical (that is eccentricity of Tethys to be 0.0008 ) and chaos was taken into account. It means probability of capture in $i_{1}^{2}$ or $i_{3}^{2}$ resonance is 0.24 (i.e. probability of non capture in $i_{1} i_{3}$ resonance). Here we have done the comparative study of the dynamics of Mimas-Tethys system at $i_{1} i_{3}, i_{1} i_{3} e_{3}, i_{1}^{2} e_{3}, i_{3}^{2} e_{3}$ and

$i_{1}^{2}, i_{1} i_{3} e_{3}, i_{1}^{2} e_{3}, i_{3}^{2} e_{3}$ resonances along with secular resonance of Saturn's six inner satellites and Saturn's oblateness. We have drawn Poincare surface of sections and Time series graphs to compare their effect.
\end{abstract}

\section{Keywords}

Secular Resonance, Three Body Problem, Disturbing Function, Oblateness

\section{Introduction}

Allan [1] and Sinclair [2] investigated the dynamics of the Mimas-Tethys system under the hypothesis of circular orbits. Allan found (backward in time) the values for the satellites inclinations before capture in the present resonance to be $i_{1}=0.42^{\circ}$ and $i_{3}=1.05^{\circ}$ and Sinclair found the probability of capture to be 0.04 .

Vienne and Duriez [3] discovered, using the frequency analysis method developed by Laskar [4], a particularly interesting $200 \mathrm{yr}$ period in the mean longitude of Mimas which came because oblateness of Saturn and the interaction between Mimas, Enceladus, Tethys, Dione, Rhea and Titan (See [4] [5] [6] [7]) set the eccentricity of Tethys 0.000235 , but with some uncertainties, it could amount to 0.001 . This is a very small value, which certainly explains why the previous studies would assume Tethys moving on a circular orbit. However, Champenois 
and Vienne showed that in spite of such a small value, the third order terms with arguments $\psi+\varphi=3 \lambda_{1}-6 \lambda_{3}+2 \Omega_{1}+\varpi_{3}$, $\psi=\lambda_{1}-2 \lambda_{3}+\Omega_{1}-\Omega_{3}+\varpi_{3}, \quad \psi-\varphi=-\lambda_{1}+2 \lambda_{3}-2 \Omega_{3}+\varpi_{3}$ and $\varphi=2 \lambda_{1}-4 \lambda_{3}+\Omega_{1}+\Omega_{3}$ brought chaos in the dynamics of Mimas-Tethys system because of near vanishing frequency $n_{1}-2 n_{3}+\dot{\Omega}_{1}-\dot{\Omega}_{3}+\dot{\varpi}_{3}$.

Greenberg [8] analyzed the effect of $i_{1}^{2}$ and $i_{3}^{2}$ resonances on Mimas-Tethys system along with $i_{1} i_{3}$ resonance.

Champenois and Vienne [5] [6] numerically investigated the role of 200 year period and found that the inclination of Mimas before capture might have been higher (up to 0.7 degree) or lower (down to 0.03 degree) than that of previously considered 0.42 degree. Also Tethys's eccentricity on capture may have been quite higher ( 0.0008 versus 0 ). This value of eccentricity was found by a method which took chaos into account. They also found that probability of capture in $i_{1} i_{3}$ resonance was 0.76 when eccentricity of Tethys was 0.0008 .

Jha and Agrawal [9] [10] have done the comparative study of the dynamics of Mimas-Tethys at $i_{1}^{2},, i_{1} i_{3}$ and $i_{3}^{2}$ resonances along with three third order resonances with and without considering the secular term of all inner satellites and oblateness of Saturn. Jha and Jha [11] [12] [13] [14] have studied the secular resonance effect of Dione, Rhea and Titan on this system.

Thomas, P. C. et al. [15] have measured the shapes and sizes of six icy satellites by Cassini imaging subsystem (ISS) data, employing limb coordinates and stereogrammetic control points. Mimas, Enceladus, Tethys, Dione and Rhea are all well described by triaxial ellipsoids; Iapetus is best represented by an oblate spheroid.

Czechowski et al. [16] found that the temperature of Mimas interior was significantly lower than that of Enceladus. Czechowski and Losiak [17] have investigated the early thermal history of Rhea and have found that liquid state convection could delay the differentiation for hundreds of millions years.

The physical model was taken to be Mimas and Tethys on eccentric orbits inclined on the equatorial plane of the Saturn. Saturn's gravitational momenta are essential as they provide the main contribution to the orbital precession rates and we had to take into account the lowest degree oblateness terms

$J_{2}, J_{4}, J_{2}^{2}, J_{6}, J_{2} J_{4}$ and $J_{2}^{3}$ (see Table 1 for its values), also the actions of the Japet in the equations whereas the Sun and the small satellites of Saturn are not taken into account because of their weak effects in the generations of the perturbative frequency $\dot{\sigma}$.

Here the notations $a_{1}, n_{1}, e_{1}, i_{1}, \gamma_{1}, \varpi_{1}, \Omega_{1}$ and $\lambda_{1}$ are orbital semi-major axis, mean motion, eccentricity, inclination, sine of semi inclination, longitudes of periapse, longitude of ascending node and mean longitude of Mimas respectively. Corresponding notions with subscript 2, 3, 4, 5 and 6 refers to the Enceladus, Tethys, Dione, Rhea and Titan's orbital elements. Small $m_{1}, m_{2}, m_{3}, m_{4}, m_{5}$ and $m_{6}$ stands for Mimas, Enceladus, Tethys, Dione, Rhea and Titan's masses with unit of Saturn.

Here we are extending the work of Jha and Agrawal [9] in $i_{1}^{2}$ and $i_{1} i_{3}$ resonance by considering the effect of secular term of all inner satellites along with 
Table 1. Parameters of the three-body system Mimas, Enceladus, Tethys, Dione, Rhea, Titan and Saturn $M_{i, i=1-6}, \quad M$ and $M_{S}$ are the masses of the considered satellite, Saturn and the Sun, respectively.

\begin{tabular}{cccccccccc}
\hline & $m=\frac{M_{i, i=1-6}}{M}$ & $n(\mathrm{rad} / \mathrm{yr})$ & $i(\mathrm{deg})$ & $M_{s} / M$ & ae $(\mathrm{km})$ & $E$ & $J_{2}$ & $J_{4}$ & $J_{6}$ \\
\hline Mimas & $6.34 \times 10^{-8}$ & 2422.44 & 1.62 & - & - & 0.0194 & & \\
Enceladus & $0.15 \times 10^{-6}$ & & & & & & & \\
Tethys & $1.06 \times 10^{-6}$ & 1213.17 & 1.093 & - & - & 0.009 & & \\
Dione & $1.963 \times 10^{-6}$ & & & & & & & \\
Rhea & $4.32 \times 10^{-6}$ & - & - & - & - & - & & \\
Titan & $236.638 \times 10^{-6}$ & & & & & & & \\
Saturn & - & - & - & 3498.79 & 60330 & - & 0.01298 & 0.000915 & 0.000095 \\
\hline
\end{tabular}

Saturn Oblateness.

\section{Equation of Motion When the System Is Locked in $i_{1}{ }^{2}$, $i_{1}^{2} e_{3}, \quad i_{1} i_{3} e_{3}$ and $i_{3}^{2} e_{3}$ Resonances}

(Equations of motion is taken from [9])

$$
\begin{gathered}
\ddot{v}=A_{v} \operatorname{Sin}[v]+A_{\psi+\varphi} \operatorname{Sin}[\psi+\varphi]+A_{\psi} \operatorname{Sin}[\psi]+A_{\psi-\varphi} \operatorname{Sin}[\psi-\varphi] \\
=A_{v}\left[\operatorname{Sin}(v)+A_{v 1} \operatorname{Sin}\left(\frac{3}{2} v-\frac{3}{2}\left(\Omega_{1}-\Omega_{3}\right)+\sigma\right)\right. \\
\left.+A_{v 2} \operatorname{Sin}\left(\frac{1}{2} v-\frac{1}{2}\left(\Omega_{1}-\Omega_{3}\right)+\sigma\right)+A_{v 3} \operatorname{Sin}\left(-\frac{1}{2} v+\frac{1}{2}\left(\Omega_{1}-\Omega_{3}\right)+\sigma\right)\right] \\
v=2 \lambda_{1}-4 \lambda_{3}+2 \Omega_{1}, \\
\psi+\varphi=3 \lambda_{1}-6 \lambda_{3}+2 \Omega_{1}+\varpi_{3}, \\
\psi+\varphi=\frac{3}{2} v-\frac{3}{2}\left(\Omega_{1}-\Omega_{3}\right)+\sigma,
\end{gathered}
$$

where,

$$
\begin{aligned}
\sigma=\frac{1}{2} \Omega_{1} & -\frac{3}{2} \Omega_{3}+\varpi_{3}=f t+\sigma_{0}=\frac{2 \pi}{200} t+\sigma_{0} \\
\psi & =\lambda_{1}-2 \lambda_{3}+\Omega_{1}-\Omega_{3}+\varpi_{3} \\
& =\frac{1}{2}\left(v-\Omega_{1}+\Omega_{3}\right)+\sigma \\
& =\frac{1}{2} v-\frac{1}{2}\left(\Omega_{1}-\Omega_{3}\right)+f t+\sigma_{0}
\end{aligned}
$$

and

$$
\begin{aligned}
\psi-\varphi & =-\lambda_{1}+2 \lambda_{3}-2 \Omega_{3}+\varpi_{3} \\
& =-\frac{1}{2}\left(v-\Omega_{1}+\Omega_{3}\right)+\sigma \\
& =-\frac{1}{2} v+\frac{1}{2}\left(\Omega_{1}-\Omega_{3}\right)+f t+\sigma_{0}
\end{aligned}
$$

where $f$ is the frequency of $\sigma$ at time $t . \sigma_{0}$ is the value of $\sigma$ at time $t=0$ 
(J2000) (see [6] [7]). Here $v=\varphi-\left(\Omega_{1}-\Omega_{3}\right)$ (for values see Table 2).

$$
\begin{gathered}
A_{v}=\left(6 n_{1}^{2} m_{3} \alpha_{13}+24 n_{3}^{2} m_{1}\right)\left(-f_{0}\left(\alpha_{13}\right)\right) \gamma_{1}^{2} \\
A_{v 1}=-3 e_{3} \frac{f_{1}\left(\alpha_{13}\right)}{f_{0}\left(\alpha_{13}\right)}, A_{\nu 2}=-e_{3} \frac{f_{2}\left(\alpha_{13}\right)}{f_{0}\left(\alpha_{13}\right)} \frac{\gamma_{3}}{\gamma_{1}} \text { and } A_{\nu 3}=e_{3} \frac{f_{3}\left(\alpha_{13}\right)}{f_{0}\left(\alpha_{13}\right)} \frac{\gamma_{3}^{2}}{\gamma_{1}^{2}} . \\
A_{\psi+\varphi}=\left(18 n_{1}^{2} m_{3} \alpha_{13}+72 n_{3}^{2} m_{1}\right) e_{3} f_{1}\left(\alpha_{13}\right) \gamma_{1}^{2}=-3 A_{v} e_{3} \frac{f_{1}\left(\alpha_{13}\right)}{f_{0}\left(\alpha_{13}\right)} . \\
A_{\psi}=\left(6 n_{1}^{2} m_{3} \alpha_{13}+24 n_{3}^{2} m_{1}\right) e_{3} \gamma_{1} \gamma_{3} f_{2}\left(\alpha_{13}\right)=-A_{v} e_{3} \frac{f_{2}\left(\alpha_{13}\right)}{f_{0}\left(\alpha_{13}\right)} \frac{\gamma_{3}}{\gamma_{1}} . \\
A_{\psi-\varphi}=-\left(6 n_{1}^{2} m_{3} \alpha_{13}+24 n_{3}^{2} m_{1}\right) e_{3} \gamma_{3}^{2} f_{3}\left(\alpha_{13}\right)=A_{v} e_{3} \frac{f_{3}\left(\alpha_{13}\right)}{f_{0}\left(\alpha_{13}\right)} \frac{\gamma_{3}^{2}}{\gamma_{1}^{2}} .
\end{gathered}
$$

The value of $f_{0}\left(\alpha_{13}\right), f_{1}\left(\alpha_{13}\right), f_{2}\left(\alpha_{13}\right)$ and $f_{3}\left(\alpha_{13}\right)$ depend on the Laplace's Coefficients $b_{s}^{(k)}\left(\alpha_{i j}\right)$ and its values are given in Table 2.

\section{Equation of Motion When the System Is Locked in $i_{1} i_{3}$,}

$$
\begin{aligned}
i_{1}^{2} e_{3}, & i_{1} i_{3} e_{3} \text { and } i_{3}^{2} e_{3} \text { Resonances } \\
\frac{\mathrm{d}^{2} \varphi}{\mathrm{d} t^{2}}= & A_{0}\left(\operatorname{Sin} \varphi+A_{1} \operatorname{Sin}\left(\frac{3}{2} \varphi+f t+\sigma_{0}\right)+A_{2} \operatorname{Sin}\left(\frac{1}{2} \varphi+f t+\sigma_{0}\right)\right. \\
& \left.+A_{3} \operatorname{Sin}\left(-\frac{1}{2} \varphi+f t+\sigma_{0}\right)\right)+\left(\frac{\mathrm{d}^{2} \varphi}{\mathrm{d} t^{2}}\right)_{o}+\left(\frac{\mathrm{d}^{2} \sigma}{\mathrm{d} t^{2}}\right)_{o}+\left(\frac{\mathrm{d}^{2} \varphi}{\mathrm{d} t^{2}}\right)_{s}+\left(\frac{\mathrm{d}^{2} \sigma}{\mathrm{d} t^{2}}\right)_{s}
\end{aligned}
$$

where

$$
\begin{gathered}
\varphi=2 \lambda_{1}-4 \lambda_{3}+\Omega_{1}+\Omega_{3}, \\
\psi+\varphi=3 \lambda_{1}-6 \lambda_{3}+2 \Omega_{1}+\varpi_{3} \\
=\frac{3}{2} \varphi+\sigma \\
\psi=\lambda_{1}-2 \lambda_{3}+\Omega_{1}-\Omega_{3}+\varpi_{3} \\
=\frac{1}{2} \varphi+\sigma
\end{gathered}
$$

and

Table 2. Analytical expressions of the functions $f_{i}\left(\alpha_{13}\right)$ for the arguments $\varphi, \psi+\varphi, \psi, \psi-\varphi$ and $v$ with their value for $\alpha_{13}=0.6306386$ (TASS1.6 [7]).

\begin{tabular}{cccc}
\hline Argument & $\mathrm{I}$ & $f_{i}(\alpha)$ & $f_{i}\left(\alpha_{13}\right)$ \\
\hline$\varphi$ & 0 & $-\alpha_{13} b_{3 / 2}^{3}\left(\alpha_{13}\right)$ & -1.65088068 \\
$\psi+\varphi$ & 1 & $3 \alpha_{13} b_{3 / 2}^{4}\left(\alpha_{13}\right)+\frac{1}{4}\left(\alpha_{13}\right)^{2} \frac{\mathrm{d}}{\mathrm{d} \alpha} b_{3 / 2}^{4}\left(\alpha_{13}\right)$ & 5.23786953 \\
$\psi$ & 2 & $2 \alpha_{13} b_{3 / 2}^{2}\left(\alpha_{13}\right)+\frac{1}{2}\left(\alpha_{13}\right)^{2} \frac{\mathrm{d}}{\mathrm{d} \alpha} b_{3 / 2}^{2}\left(\alpha_{13}\right)$ & 9.70821605 \\
$\psi-\varphi$ & 3 & $-\alpha_{13} b_{3 / 2}^{2}\left(\alpha_{13}\right)+\frac{1}{4}\left(\alpha_{13}\right)^{2} \frac{\mathrm{d}}{\mathrm{d} \alpha} b_{3 / 2}^{2}\left(\alpha_{13}\right)$ & 0.22188903 \\
$v$ & 4 & $\frac{1}{2} \alpha_{13} b_{3 / 2}^{3}\left(\alpha_{13}\right)=-f_{0}\left(\alpha_{13}\right)$ & 0.82544034 \\
\hline
\end{tabular}




$$
\begin{aligned}
\psi-\varphi & =-\lambda_{1}+2 \lambda_{3}-2 \Omega_{3}+\varpi_{3} \\
& =-\frac{1}{2} \varphi+\sigma .
\end{aligned}
$$

With $\sigma=f t+\sigma_{0}$ where $\sigma_{0}=-0.031391995$ and

$$
\begin{gathered}
A_{0}=\left(12 n_{1}^{2} m_{3} \alpha_{13}+48 n_{3}^{2} m_{1}\right) f_{0}\left(\alpha_{13}\right) \gamma_{1} \gamma_{3} \\
A_{1}=\frac{3}{2} e_{3} \frac{f_{1}\left(\alpha_{13}\right)}{f_{0}\left(\alpha_{13}\right)} \frac{\gamma_{1}}{\gamma_{3}}, A_{2}=\frac{1}{2} e_{3} \frac{f_{2}\left(\alpha_{13}\right)}{f_{0}\left(\alpha_{13}\right)} \text { and } A_{3}=-\frac{1}{2} \frac{f_{3}\left(\alpha_{13}\right)}{f_{0}\left(\alpha_{13}\right)} \frac{\gamma_{3}}{\gamma_{1}} .
\end{gathered}
$$

The values of $f_{0}\left(\alpha_{13}\right), f_{1}\left(\alpha_{13}\right), f_{2}\left(\alpha_{13}\right)$ and $f_{3}\left(\alpha_{13}\right)$ depend on the Laplace's Coefficients $b_{s}^{(k)}\left(\alpha_{i j}\right)$ and given in Table 2.

With

$$
\begin{aligned}
& \left(\frac{\mathrm{d}^{2} \lambda_{1}}{\mathrm{~d} t^{2}}\right)_{S}=-2\left[\sum_{i=2}^{5} m_{i} \alpha_{1 i}^{2} \frac{\mathrm{d}^{2} A\left(\alpha_{1 i}\right)}{\mathrm{d} \alpha_{1 i}^{2}}\right] \frac{\mathrm{d} n_{1}}{\mathrm{~d} t} \\
& \left(\frac{\mathrm{d}^{2} \Omega_{1}}{\mathrm{~d} t^{2}}\right)_{S}=\frac{1}{2}\left[\sum_{i=2}^{5} m_{i} \alpha_{1 i} C\left(\alpha_{1 i}\right)\right] \frac{\mathrm{d} n_{1}}{\mathrm{~d} t} \\
& \left(\frac{\mathrm{d}^{2} \Omega_{3}}{\mathrm{~d} t^{2}}\right)_{S}=\frac{1}{2}\left[\sum_{i=1}^{2} m_{i} C\left(\alpha_{i 3}\right)+\sum_{i=4}^{6} m_{i} \alpha_{3 i} C\left(\alpha_{3 i}\right)\right] \frac{\mathrm{d} n_{3}}{\mathrm{~d} t} \\
& \left(\frac{\mathrm{d}^{2} \varpi_{3}}{\mathrm{~d} t^{2}}\right)_{S}=2\left[\sum_{i=1}^{2} m_{i} B\left(\alpha_{i 3}\right)+\sum_{i=4}^{6} m_{i} \alpha_{3 i} B\left(\alpha_{3 i}\right)\right] \frac{\mathrm{d} n_{3}}{\mathrm{~d} t} \\
& \left(\frac{\mathrm{d}^{2} \lambda_{3}}{\mathrm{~d} t^{2}}\right)_{S}=2\left[\sum_{i=1}^{2}-m_{i}\left\{\alpha_{i 3} \frac{\mathrm{d} A\left(\alpha_{i 3}\right)}{d \alpha_{i 3}}+A\left(\alpha_{i 3}\right)\right\}+\sum_{i=4}^{6} m_{i} \alpha_{3 i}{ }^{2} \frac{\mathrm{d} A\left(\alpha_{3 i}\right)}{\mathrm{d} \alpha_{3 i}}\right] \frac{\mathrm{d} n_{3}}{\mathrm{~d} t}
\end{aligned}
$$

(We are not considering any changes in semi major axis of any satellites) and

$$
\begin{aligned}
& \left(\frac{\mathrm{d}^{2} \varphi}{\mathrm{d} t^{2}}\right)_{S}=\left(\frac{\mathrm{d}^{2} \lambda_{1}}{\mathrm{~d} t^{2}}\right)_{S}-2\left(\frac{\mathrm{d}^{2} \lambda_{3}}{\mathrm{~d} t^{2}}\right)_{S}+\frac{1}{2}\left[\left(\frac{\mathrm{d}^{2} \Omega_{1}}{\mathrm{~d} t^{2}}\right)_{S}+\left(\frac{\mathrm{d}^{2} \Omega_{3}}{\mathrm{~d} t^{2}}\right)_{S}\right] \\
& \left(\frac{\mathrm{d}^{2} \sigma}{\mathrm{d} t^{2}}\right)_{S}=\frac{1}{2}\left(\frac{\mathrm{d}^{2} \Omega_{1}}{\mathrm{~d} t^{2}}\right)_{S}-\frac{3}{2}\left(\frac{\mathrm{d}^{2} \Omega_{3}}{\mathrm{~d} t^{2}}\right)_{S}+\left(\frac{\mathrm{d}^{2} \varpi_{3}}{\mathrm{~d} t^{2}}\right)_{S} .
\end{aligned}
$$

Now we will find the terms due to Oblateness of Saturn. Saturn's gravitational momenta are quite important so that we have, in order to get the full variations of the mean longitudes, nodes and pericentres due to the oblateness, taken into account the lowest-degree terms with $J_{2}, J_{4}, J_{2}^{2}, J_{6}, J_{2} J_{4}$ and $J_{2}^{3}$ (See Table 1 for its values) as a factor (Vienne and Duriez [3] [5]). The other terms are taken constant. Values of $\alpha_{i j}, A\left(\alpha_{i j}\right), B\left(\alpha_{i j}\right), C\left(\alpha_{i j}\right)$ and $\frac{\mathrm{d} A\left(\alpha_{i j}\right)}{\mathrm{d} \alpha_{i j}}$ for every pair $(i, j),(i<j)$ involving Mimas, Enceladus, Tethys, Dione, Rhea and Titan are given in Table 3.

We then get ( $a_{e}$ is the equatorial radius of Saturn).

$$
\begin{aligned}
\left(\frac{\mathrm{d}^{2} \Omega_{3}}{\mathrm{~d} t^{2}}\right)_{0}= & {\left[\frac{-3}{2} J_{2}\left(\frac{a_{e}}{a_{3}}\right)^{2}\left(1+2 e_{3}^{2}-2 \gamma_{3}^{2}\right)+a_{3}^{-4}\left(\frac{15}{4} J_{4}-\frac{45}{8} J_{2}^{2}\right)\right] \frac{\mathrm{d} n_{3}}{\mathrm{~d} t} } \\
& -6 J_{2} n_{3}\left(\frac{a_{e}}{a_{3}}\right)^{2}\left(e_{3} \frac{\mathrm{d} e_{3}}{\mathrm{~d} t}-\gamma_{3} \frac{\mathrm{d} \gamma_{3}}{\mathrm{~d} t}\right) .
\end{aligned}
$$


Table 3. Value of $\alpha_{i j}, A\left(\alpha_{i j}\right), B\left(\alpha_{i j}\right), C\left(\alpha_{i j}\right)$ and $\frac{\mathrm{d} A\left(\alpha_{i j}\right)}{\mathrm{d} \alpha_{i j}}$ for every pair $(i, j),(i<j)$ involving Mimas, Enceladus, Tethys, Dione, Rhea and Titan $\left(\alpha_{i j}\right)$ (CV [6]).

\begin{tabular}{cccccc}
\hline$i-j$ & $\alpha_{i j}$, & $A\left(\alpha_{i j}\right)$ & $B\left(\alpha_{i j}\right)$ & $C\left(\alpha_{i j}\right)$ & $\frac{\mathrm{d} A\left(\alpha_{i j}\right)}{\mathrm{d} \alpha_{i j}}$ \\
\hline $1-2$ & 0.78026 & 1.2473 & 1.3674 & -5.4695 & 1.0996 \\
$1-3$ & 0.63064 & 1.1306 & 0.38952 & -1.5581 & 0.55451 \\
$1-4$ & 0.49258 & 1.0706 & 0.15366 & -0.61463 & 0.33609 \\
$1-5$ & 0.35283 & 1.0335 & 0.059940 & -0.23976 & 0.20479 \\
$1-6$ & 0.15223 & 1.0059 & 0.0090810 & -0.036324 & 0.078148 \\
$2-3$ & 0.80824 & 1.2807 & 1.8535 & -7.4138 & 1.2978 \\
$3-4$ & 0.78108 & 1.2482 & 1.3790 & -5.5159 & 1.1047 \\
$3-5$ & 0.55948 & 1.0960 & 0.23856 & -0.95425 & 0.42538 \\
$3-6$ & 0.24140 & 1.0151 & 0.024460 & -0.097840 & 0.12912 \\
\hline
\end{tabular}

$$
\begin{aligned}
&\left(\frac{\mathrm{d}^{2} \lambda_{1}}{\mathrm{~d} t^{2}}\right)_{0}= {\left[3 J_{2}\left(\frac{a_{e}}{a_{1}}\right)^{2}\left(1+\frac{7}{4} e_{1}^{2}-7 \gamma_{1}^{2}\right)+a_{1}^{-4}\left(-\frac{15}{4} J_{4}+\frac{160}{11} J_{2}^{2}\right)\right] \frac{\mathrm{d} n_{1}}{\mathrm{~d} t} } \\
&-42 J_{2} n_{1} \gamma_{1}\left(\frac{a_{e}}{a_{1}}\right)^{2} \frac{\mathrm{d} \gamma_{1}}{\mathrm{~d} t} . \\
&\left(\frac{\mathrm{d}^{2} \lambda_{3}}{\mathrm{~d} t^{2}}\right)_{O}= {\left[3 J_{2}\left(\frac{a_{e}}{a_{3}}\right)^{2}\left(1+\frac{7}{4} e_{3}^{2}-7 \gamma_{3}^{2}\right)+a_{3}^{-4}\left(-\frac{15}{4} J_{4}+\frac{160}{11} J_{2}^{2}\right)\right] \frac{\mathrm{d} n_{3}}{\mathrm{~d} t} } \\
&-42 J_{2} n_{3} \gamma_{3}\left(\frac{a_{e}}{a_{3}}\right)^{2} \frac{\mathrm{d} \gamma_{3}}{\mathrm{~d} t} . \\
&\left(\frac{\mathrm{d}^{2} \varphi}{\mathrm{d} t^{2}}\right)_{0}=\left(\frac{\mathrm{d}^{2} \lambda_{1}}{\mathrm{~d} t^{2}}\right)_{0}-2\left(\frac{\mathrm{d}^{2} \lambda_{3}}{\mathrm{~d} t^{2}}\right)_{0}+\frac{1}{2}\left\{\left(\frac{\mathrm{d}^{2} \Omega_{1}}{\mathrm{~d} t^{2}}\right)_{0}+\left(\frac{\mathrm{d}^{2} \Omega_{3}}{\mathrm{~d} t^{2}}\right)_{0}\right\} . \\
&\left(\frac{\mathrm{d}^{2} \sigma}{\mathrm{d} t^{2}}\right)_{0}=\frac{1}{2}\left(\frac{\mathrm{d}^{2} \Omega_{1}}{\mathrm{~d} t^{2}}\right)_{0}-\frac{3}{2}\left(\frac{\mathrm{d}^{2} \Omega_{3}}{\mathrm{~d} t^{2}}\right)_{0}+\left(\frac{\mathrm{d}^{2} \varpi_{3}}{\mathrm{~d} t^{2}}\right)_{0} .
\end{aligned}
$$

\section{Numerical Integration and Surface of Sections}

Our equations were integrated backwards in time. The initial conditions are taken from TASS1.6 [7] for J2000. $i_{1}=1.62^{\circ}, i_{3}=1.093^{\circ}$ and $e_{3}=0.0008$ are taken to be fixed. Here we have integrated it for $-62.8 \times 14000$ yrs .

Figure 1 and Figure 2 are Poincare surface of sections with and without oblateness of Saturn respectively at $i_{1}^{2}, i_{1}^{2} e_{3}, i_{1} i_{3} e_{3}$ and $i_{3}^{2} e_{3}$ resonances. Figure 3 and Figure 4 are the time-series graph for the same. Figure 5 and Figure 6 are the Poincare surface of sections with and without oblateness of Saturn at $i_{1} i_{3}, i_{1}^{2} e_{3}, i_{1} i_{3} e_{3}$ and $i_{3}^{2} e_{3}$ resonances and Figure 7 and Figure 8 are the time series graph for the same.

\section{Discussion}

Vienne and Duriez [3] discovered the 200 year period in the mean longitude of 


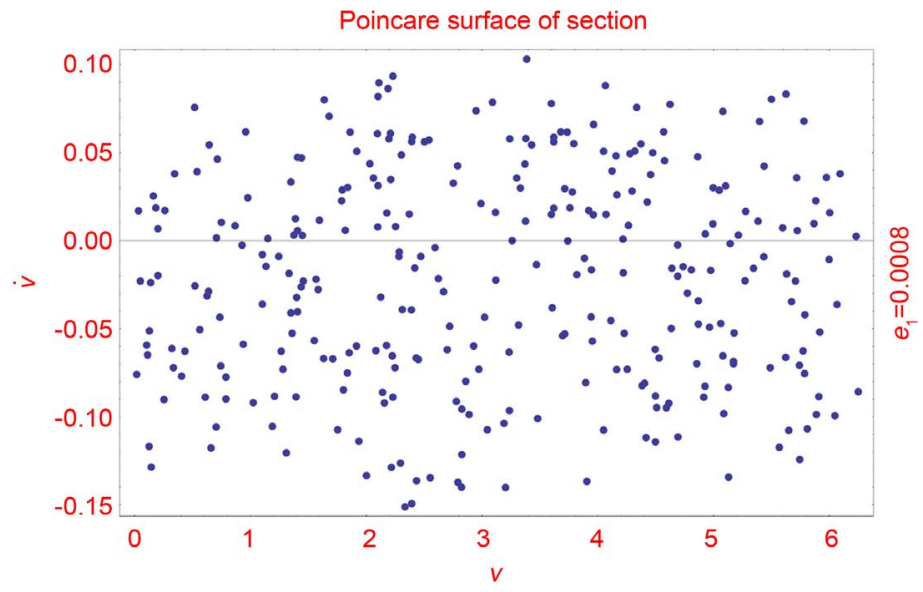

Figure 1. Poincare surface of section at $i_{1}^{2}, i_{1}^{2} e_{3}, i_{1} i_{3} e_{3}$ and $i_{3}^{2} e_{3}$ resonances with oblateness of Saturn. Secular resonances of all inner satellites have been considered at $i_{1}=1.62^{\circ}, i_{3}=1.093^{\circ}$ and $e_{3}=0.0008$.

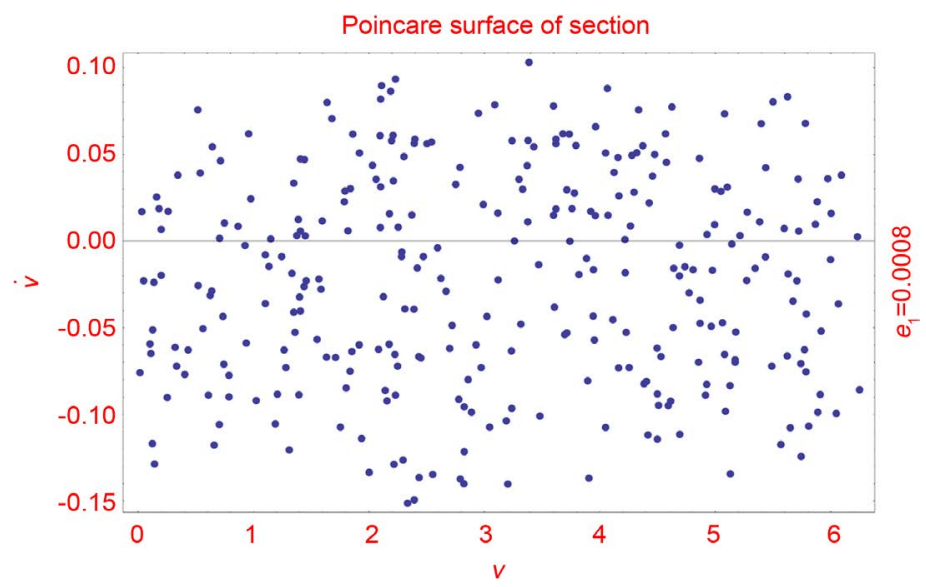

Figure 2. Poincare surface of section at $i_{1}^{2}, i_{1}^{2} e_{3}, i_{1} i_{3} e_{3}$ and $i_{3}^{2} e_{3}$ resonances without oblateness of Saturn. Secular resonances of all inner satellites have been considered at $i_{1}=1.62^{\circ}, i_{3}=1.093^{\circ}$ and $e_{3}=0.0008$.

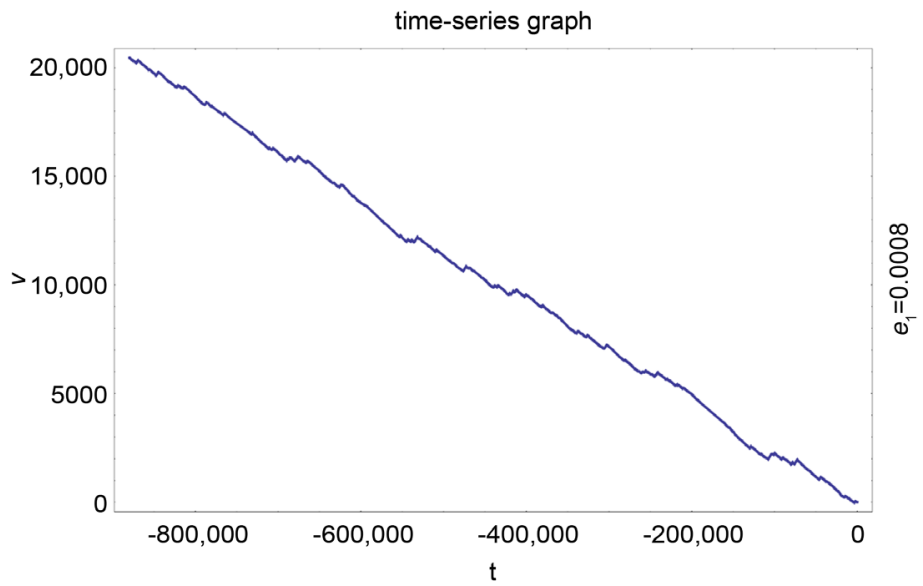

Figure 3. Time-series graph at $i_{1}^{2}, i_{1}^{2} e_{3}, i_{1} i_{3} e_{3}$ and $i_{3}^{2} e_{3}$ resonances with oblateness of Saturn. Secular resonances of all inner satellites have been considered at $i_{1}=1.62^{\circ}, i_{3}=1.093^{\circ}$ and $e_{3}=0.0008$. 


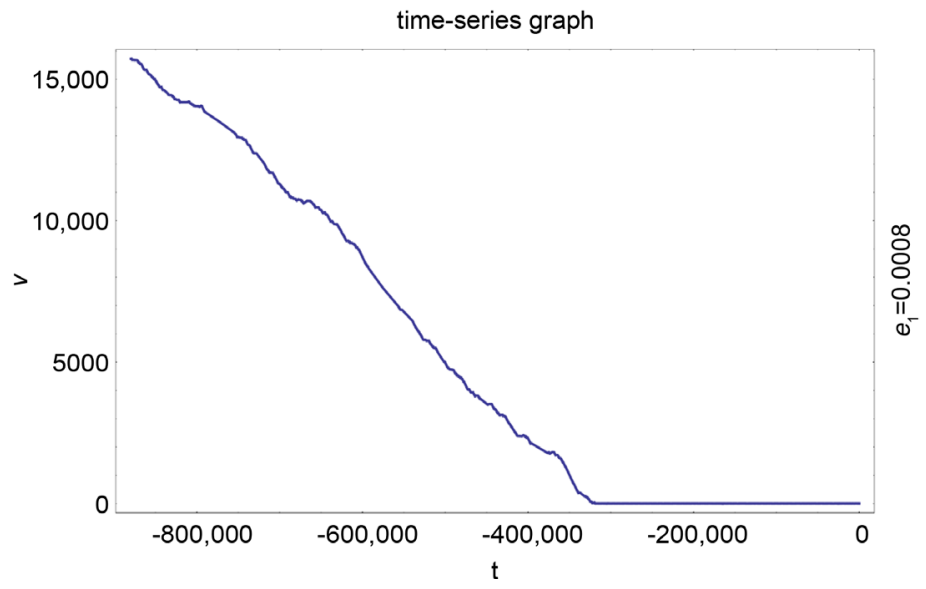

Figure 4. Time-series graph at $i_{1}^{2}, i_{1}^{2} e_{3}, i_{1} i_{3} e_{3}$ and $i_{3}^{2} e_{3}$ resonances without oblateness of Saturn. Secular resonances of all inner satellites have been considered at $i_{1}=1.62^{\circ}, i_{3}=1.093^{\circ}$ and $e_{3}=0.0008$.

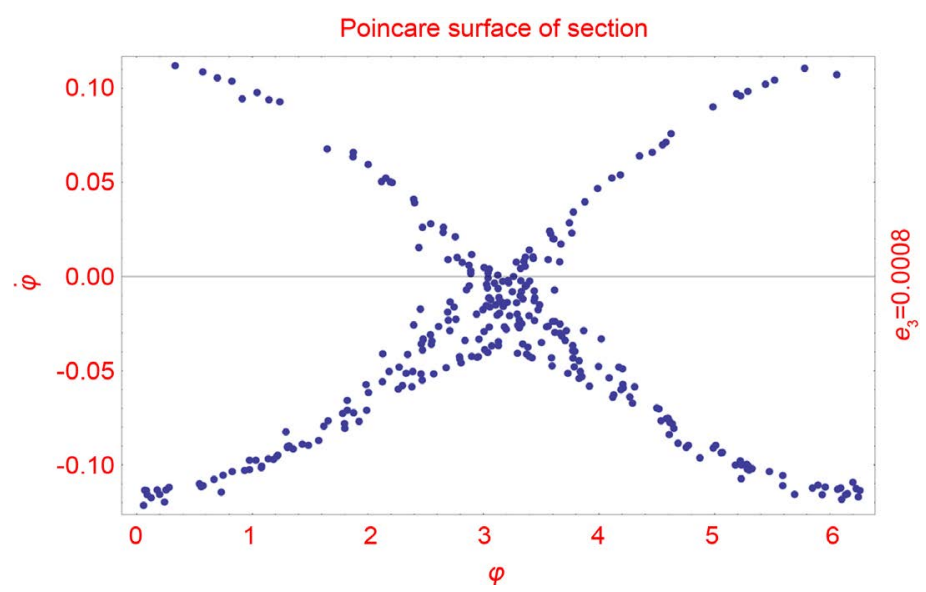

Figure 5. Poincare surface of section at $i_{1} i_{3}, i_{1}^{2} e_{3}, i_{1} i_{3} e_{3}$ and $i_{3}^{2} e_{3}$ resonances with oblateness of Saturn. Secular resonances of all inner satellites have been considered at $i_{1}=1.62^{\circ}, i_{3}=1.093^{\circ}$ and $e_{3}=0.0008$.

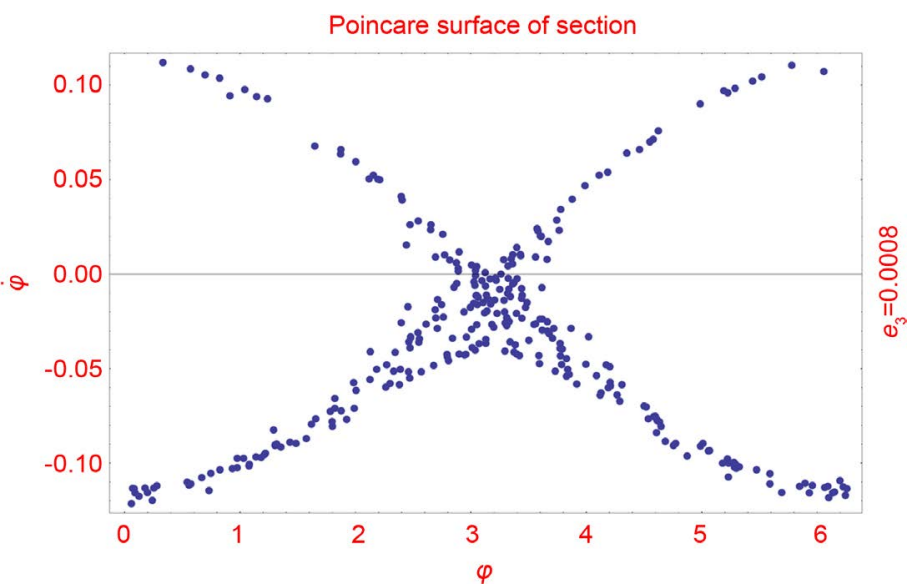

Figure 6. Poincare surface of section at $i_{1} i_{3}, i_{1}^{2} e_{3}, i_{1} i_{3} e_{3}$ and $i_{3}^{2} e_{3}$ resonances without oblateness of Saturn. Secular resonances of all inner satellites have been considered at $i_{1}=1.62^{\circ}, i_{3}=1.093^{\circ}$ and $e_{3}=0.0008$. 


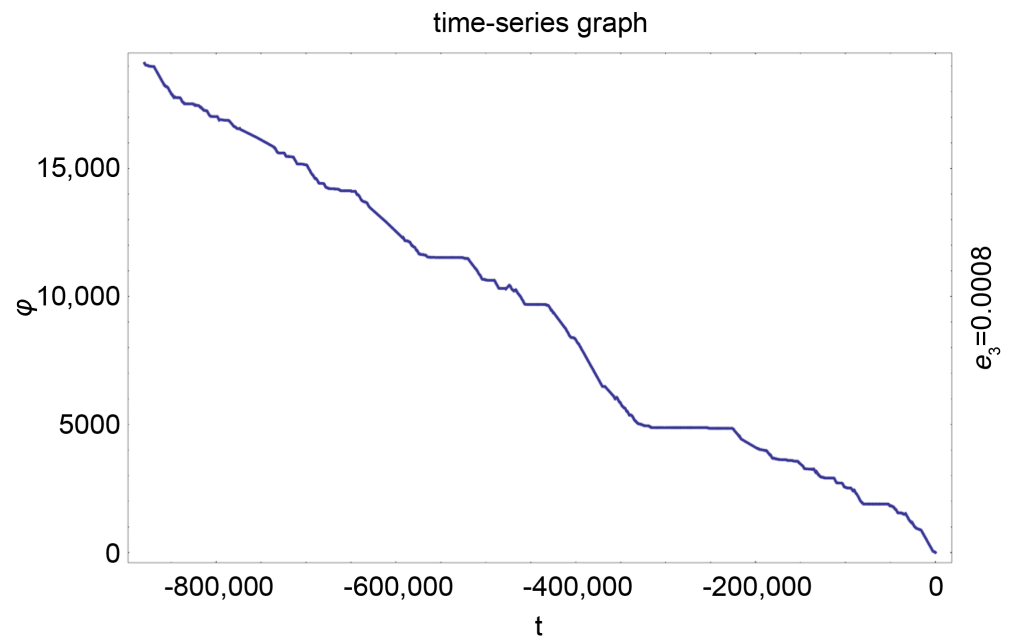

Figure 7. Time-series graph at $i_{1} i_{3}, i_{1}^{2} e_{3}, i_{1} i_{3} e_{3}$ and $i_{3}^{2} e_{3}$ resonances withoblateness of Saturn. Secular resonances of all inner satellites have been considered at $i_{1}=1.62^{\circ}, i_{3}=1.093^{\circ}$ and $e_{3}=0.0008$.

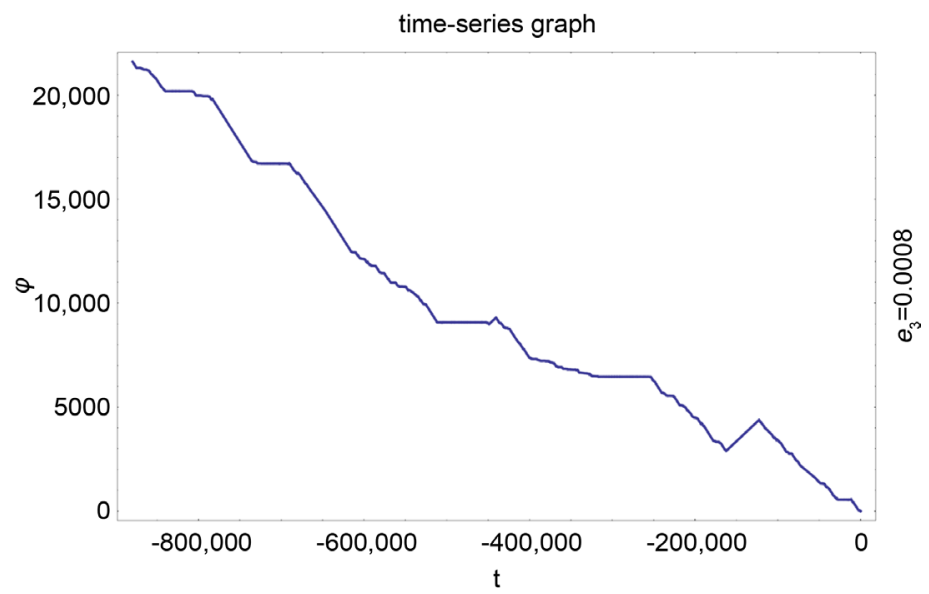

Figure 8. Time-series graph at $i_{1} i_{3}, i_{1}^{2} e_{3}, i_{1} i_{3} e_{3}$ and $i_{3}^{2} e_{3}$ resonances without oblateness of Saturn. Secular resonances of all inner satellites have been considered at $i_{1}=1.62^{\circ}, i_{3}=1.093^{\circ}$ and $e_{3}=0.0008$.

Mimas. Champenois and Vienne [5] [6] have investigated the role of 200 year long period on the dynamics of Mimas-Tethys system when considered to be presently trapped in $i_{1} i_{3}$ resonance with probability of capture 0.76 at 2:4 commensurability. They found that the sources of this period were both the oblateness of Saturn and the interaction between its six inner satellites. They also realized that considering an eccentric orbit of Tethys upseted the earlier vision of dynamics of the Mimas-Tethys system.

Here, we have analyzed that the system is more chaotic if considered to be (at presently it is) locked in $i_{1} i_{3}$ resonance compared with $i_{1}^{2}$ resonance; we consider the effect of Saturns oblateness and the interaction of its six inner satellites, by the help of Poincare surface of section and time-series graphs which confirm the earlier results too. Figure 2 and Figure 6 are Poincare surface of sections and Figure 4 and Figure 8 are time series graphs without Oblateness of Saturn. Al- 
though we could not quantify the chaos, on the basis of above figures, we can say that oblateness of Saturn plays a significant role in the dynamics of Mimas-Tethys system and also it partially controls the chaos.

\section{References}

[1] Allan, R.R. (1969) Evolution of Mimas-Tethys Commensurabilities. Astronomical Journal, 74, 997-506. https://doi.org/10.1086/110827

[2] Sinclair, A.T. (1972) On the Origin of the Commensurabilities amongst the Satellites of Saturn. Monthly Notices of the Royal Astronomical Society, 160, 169-187. https://doi.org/10.1093/mnras/160.2.169

[3] Vienne, A. and Duriez, L. (1992) A General Theory of Motion for the Eight Major Satellites of Saturn. III. Long-Period Perturbations. Astronomy and Astrophysics, 257, 331-352.

[4] Laskar, J. (1990) The Chaotic Motion of the Solar System: A Numerical Estimate of the Size of the Chaotic Zones. Icarus, 88, 266-291.

[5] Vienne, A. and Duriez, L. (1995) TASS1.6: Ephimerides of the Major Saturnian Satellites. Astronomy and Astrophysics, 297, 588-605.

[6] Champenois, S. and Vienne, A. (1999) The Role of Secondary Resonances in the Evolution of the Mimas-Tethys System. Icarus, 140, 106-121.

[7] Champenois, S. and Vienne, A. (1999) Chaos and Secondary Resonances in the Mimas-Tethys System. Celestial Mechanics and Dynamical Astronomy, 74, 111-146. https://doi.org/10.1023/A:1008314007365

[8] Greenberg, R. (1973) The Inclination-Type Resonance of Mimas and Tethys. Monthly Notices of the Royal Astronomical Society, 165, 305-311. https://doi.org/10.1093/mnras/165.3.305

[9] Jha, S. and Agrawal, S.K. (2014) Effect of Second and Third Order Resonances on Mimas-Tethys System. IJAMM, 3, 9-21.

[10] Jha, S. and Agrawal, S.K. (2014) Chaotic Dynamics of Mimas-Tethys System Due to Secular Resonance of Enceladus. GJDSA, 4, 1-11.

[11] Jha, G.K. and Jha, S. (2015) Comparative Study of Secular Resonance Effect of Enceladus and Dione on Mimas-Tethys System. IJAMM, 4, 27-31.

[12] Jha, G.K. and Jha, S. (2016) Combined Effect of Oblateness of Saturn and Secular Resonance of Rhea on Mimas-Tethys System. $M_{0}$, 3. (Accepted)

[13] Jha, G.K. and Jha, S. (2016) Role of Saturn's Oblateness and Secular Resonance of Va-rious Combination of Inner Satellites with Titan on Mimas-Tethys System. IJST. (Accepted)

[14] Jha, G.K. and Jha, S. (2017) Comparative Study of the Dynamics of Mimas-Tethys System at Different Resonances. International Journal of Advance Astronomy, 5, 44-49. (Accepted) https://doi.org/10.14419/ijaa.v5i1.7095

[15] Thomas, P.C., et al. (2007) Shapes of the Saturninan Icy Satellites and Their Significance. Icarus, 190, 573-584.

[16] Czechowski, L. and Witek, P.P. (2015) Camparison of Early Evolution of Mimas and Enceladus. Acta Geophysica, 63, 900-921. https://doi.org/10.1515/acgeo-2015-0024

[17] Czechowski, L. and Losiak, A. (2016) Early thermal History of Rhea: The Role of Serpentinization and Liquid State Convection. Acta Geophysica, 64, 2677-2716. https://doi.org/10.1515/acgeo-2016-0064 
Submit or recommend next manuscript to SCIRP and we will provide best service for you:

Accepting pre-submission inquiries through Email, Facebook, LinkedIn, Twitter, etc. A wide selection of journals (inclusive of 9 subjects, more than 200 journals)

Providing 24-hour high-quality service

User-friendly online submission system

Fair and swift peer-review system

Efficient typesetting and proofreading procedure

Display of the result of downloads and visits, as well as the number of cited articles Maximum dissemination of your research work

Submit your manuscript at: http://papersubmission.scirp.org/

Or contact ijaa@scirp.org 